ALTAMIRANO, G. REYES y F. CONDE 1995a- Check-list of Andalucía (S. Spain) seaweeds. I. Phaeophyceae. Acta Bot. Malacitana, 20: 5-18.

FLORES MOYA, A. J. SOTO, A. SANCHEZ, M. ALTAMIRANO, G. REYES y F.CONDE 1995b- Check-list of Andalucía (S. Spain) seaweeds. II. Chlorophyceae. Acta Bot. Malacitana, 20: 19-26.

GAllardo, T., A. GomeZ GARRETA, M. A. RIBERA, M. ALVAREZ y F.CONDE - 1985- A preliminary checklist of Iberian benthic marine algae. Real Jardín Botánico. Madrid. 83 pp.

GÓMEZ GARRETA, A., M. C. BARCELO, J. RULLLLUCH, M. A. RIBERA y J. ECHEGARAY. -1992Apports chorologiques du Genre Cystoseira C. Agardh. Rapp. Comm. int. Mer Médit., 33: 40.

GONZALEZ GARCIA, J. A. y F. CONDE POYALES 1994-Catálogo del macrofitobentos del Mediterráneo de Marruecos. Acta Bot. Malacitana, 19: 5-27.

IZQUIERDO, J. L., F. CONDE y A. FLORES MOYA -1995-Adiciones a la distribución geográfica del género Phyllariopsis Henry et South (Laminariales Phaeophyta) en la Península Ibérica e Islas Baleares. Acta Bot. Malacitana, 20:281-283.
MAGGS C. A. \& HOMMERSAND M. H. -1993Seaweeds of the British Isles. Vol. 1 . Rhodophyta. Part 3A. Ceramiales. British Museum: HMSO. London. 444 pp.

MIRANDA F. - 1931- Sobre las algas y Cianofíceas del Cantábrico, especialmente de Gijón. Trab. mus. Nac. Ci. Nat., Ser. Bot., 25: 1-106.

RINDI, F. \& F. CINELLI -1995-Contribution to the knowledge of the benthic algal flora of the Isle of Alboran, with notes on some little-know species in the mediterranean. Cryptogamie, Algol., 16(2): 103-114.

SOTO MORENO, J. y F. CONDE POYALES -1993Datos sobre la flora algal bentónica de la Isla de Alborán (Mar de Alborán. Mediterráneo Occidental). Cryptogamie, Algol., 14(4): 183-190.

TAYLOR, R. -1960- Marine algae of the eastern tropical and subtropical coasts of the Americas. The university of Michigan Press. 870pp.

Aceptado para su publicación en julio de 2000

Dirección de los autores. Departamento de Biología Vegetal. Facultad de Ciencias Universidad de Málaga. Apdo. 59. 29080 Málaga.

\title{
87. BRIÓFITOS NUEVOS O INTERESANTES PARA LA FLORA DE GALICIA
}

\author{
Juan REINOSO FRANCO y Aida GARCÍA MOLARES
}

Bryophytes new or rare for the flora of Galicia

Palabras clave. Briófitos, corología, flora, Galicia, España.

Key words. Bryophytes, chorology, flora, Galicia, Spain.

Se aportan nuevos datos sobre briófitos recolectados recientemente en la localidad de Salvaterra de Miño (Pontevedra), de una importancia ecológica apreciable, considerados amenazados e incluidos en la Lista Roja de los Briófitos de la Península Ibérica (Sergio et al., 1994) con la categoría de especies raras y vulnerables respectivamente. La localidad de 
Salvaterra de Miño se encuadra desde el punto de vista biogeográfico en el subsector Miñense, sector Galaico-Portugués, provincia CántabroAtlántica.

\section{Cryphaea heteromalla (Hedw.) Mohr}

Pontevedra: Salvaterra de Miño, 29TNG4060, sobre tronco de fresno en el río, 20.4.1998, A. García Molares SANT-bryo 2939.

Taxon de distribución suboceánicomediterránea. Primera cita para la provincia de Pontevedra. En Galicia solo se había citado el taxon en Santiago de Compostela (Casares, 1915; Reinoso et al., 1985).

\section{Cryphaea lamyana (Mont.) C. Müll.}

Pontevedra: Salvaterra de Miño 29TNG4060, sobre tronco de acacia en el río, 21.5.1998, A. García Molares, SANT-bryo 2908.

Taxon de distribución oceánicosubmediterránea-montana. Catalogado con la categoría de amenaza de especie vulnerable. Se trata de un taxon muy poco señalado en la Península Ibérica, sólo se conoce fuera de Galicia en Cádiz (Allorge, 1934; Allorge \& Allorge, 1945 in Casas et al., 1992), Asturias (río Eo, Vigón 1981, in Casas et al., 1992) y Salamanca (Luisier, 1915, in Casas et al., 1992). En Galicia es conocido en las localidades de Vivero (Casares, 1915), Salvaterra y Tuy (Luisier, 1918), Verín y Os Peares (Allorge, 1935), Portomouro (Casas, 1975), Caaveiro (Reinoso, 1984) y San Xusto (Reinoso, 1989, Briot. Hisp. 1231 BCB, in Casas et al., 1992).

\section{Corsinia coriandrina (Spreng.) Lindb.}

Pontevedra: Salvaterra de Miño 29TNG4060, en un talud, 30.3.1998, A. García Molares, SANTbryo 2938 .

Taxon de distribución suboceánicomediterránea. Notable cita para Galicia puesto que es la vez primera que se marca con exactitud una localidad. Sólo nos consta la referencia de Casares (1915) que cita la especie en los alrededores de Lugo y Orense. Jovet-Ast \& Bischler (1976) recorren la Península Ibérica en 1969 y en el sur recogen la especie en numerosas localidades y a su paso por Galicia recogen algunas hepáticas, señalando ( $p$. 952) su presencia en las provincias de Lugo y Orense, creemos sin duda que haciendo constar la inicial referencia de Casares.

\section{Lejeunea mandonii (Steph.) Schiff.}

Pontevedra: Salvaterra de Miño 29TNG4060, sobre roble, 21.5.1998, A. García Molares, SANTbryo 2937.

Taxon de distribución oceánica. Catalogada con la categoría de amenaza de especie rara. Se trata de la segunda cita para la provincia. Con anterioridad se conocía de La Toja (Buch, 1942), A Coruña (Jovet-Ast \& Bischler, 1976) y Caaveiro (Reinoso, 1984), como únicas localidades gallegas. Como caracteres utilizados en su identificación destacamos la presencia de cápsulas de superficie lisa, lóbulos dorsales de las hojas más suaves y de menor tamaño que en $L$. holtii, lo mismo que los anfigastros más estrechos que la especie próxima precedente con la que se puede confundir, coincidiendo con otras muestras contenidas en nuestros herbarios revisadas por el Dr. Schumacker.

\section{Pylaisia polyantha (Hedw.) B.S.G.}

Pontevedra: Salvaterra de Miño 29TNG4060, sobre acacia, 21 15.1998, A. García Molares, SANTbryo 2912 .

Taxon de distribución oceánico-mediterránea. Catalogada con la categoría de amenaza de especie rara. Notable cita para Galicia, dada como extinguida ya que no se había vuelto a citar desde hace más de ochenta años, luego de las iniciales referencias de Cambados (Casares, 1915) y alrededores de Pontevedra (Luisier, 1918).

Sematophyllum demisum (Wils.) Mitt.

Pontevedra: Salvaterra de Miño 29TNG4060, sobre rocas, 21.5.1998, A. García Molares, SANTbryo 2910.

Taxon de distribución suboceánicomediterránea. Catalogada con la categoría de amenaza de especie rara. Se cita por primera vez para Galicia. Recientemente hemos dado a conocer Sematophyllum substrumulosum como especie neófita para Galicia (Reinoso, 1998).

\section{BIBLIOGRAFÍA}

ALLORGE, P. -1934- Notes sur la flore bryologique de la Péninsule Ibérique. IX. Muscinées des provinces du Nord et du Centre de 1'Espagne. Rev. Bryol. Lichénol. 7: 249-301. Paris.

ALLORGE, V. \& P. ALLORGE -1945- La 
végétation et les groupements muscinaux des montagnes d' Algesiras. Mém. Mus. Nat. His. Nat. 21:85-116. Paris.

BUCH, H. -1942- Deux mousses critiques de la Péninsule Ibérique. Rev. Bryol. Lichénol. 13: 45-48. Paris.

CASARES, M. -1915- Enumeración y distribución geográfica de las Muscineas de la Península Ibérica. Trab. Mus. Nac. Cienc. Nat. Ser. Bot. 2: 1-179. Madrid.

CASAS, C. -1975- Brioteca Hispanica 1970. Acta Phytotax. Barc. 15:27-33. Barcelona.

CASAS,C., M. BRUGUES, R. CROS \& C. SERGIO -1992- Bryophyte Cartography. III: 101-150. Institut d'Estudis Catalans. Barcelona.

JOVET-AST \& H. BISCHLER - 1976- Hépatiques de la Péninsule Ibérique: Enumération, Notes écologiques. Rev. Bryol. Lichénol. 42: 931-987. Paris.

LUISIER, A. -1915- Fragments de Bryologie Ibérique. 8. Espèces nouvelles pour la flore espagnole. Broteria Ser. Bot. 13:154-157. Braga.

LUISIER, A. -1918- Fragments de Bryologie Ibérique. 14. Mousses de Galice. Brot. Ser. Bot. 16: 123-142. Braga.

REINOSO, J. - 1984- Contribución al conocimiento de la flora briofítica de Galicia. Briófitos de la fraga de Caaveiro, I. Musgos. Lazaroa 6: 237247. Madrid.
REINOSO, J. y J. ALVAREZ - 1984- Estudio de la flora briológica de la cuenca del río de Rojos (A Coruña). Acta Científica Compostelana 21:273284. Santiago de Compostela.

REINOSO, J. y M. SMITH - 1985- Estudio briológico de la ciudad de Santiago. Trabajos Compostelanos de Biología 12: 185-196. Santiago de Compostela.

REINOSO, J. -1998- Sematophyllum substrumulosum (Hampe) Britt. en España. Stvdia Botanica 17:131-132. Salamanca.

SERGIO, C., C. CASAS, M. BRUGUES \& R.M. CROS - 1994- Lista Vermelha dos Briofitos da Península Ibérica. Instituto da Conservaçao da Natureza. Museu, Laboratório e Jardim Botânico. Lisboa.

Aceptado para su publicación en mayo de 2000

Dirección de los autores. J. REINOSO FRANCO: Dpto. de Bioloxía Vegetal (Botánica). Facultade de Bioloxía. Universidade de Santiago de Compostela. 15706, Santiago de Compostela. A. GARCÏA MOLARES: Dpto. de Recursos Naturais e Medio Ambente. Facultade de Ciencias. Universidade de Vigo. 36200, Vigo. España.

\section{NOTAS SOBRE ORQUÍDEAS DE ALICANTE}

Manuel B. CRESPO, Joan PIERA y Michael R. LOWE

Notes on the Orchids of Alicante.

Palabras clave. Corología, flora, Orchidaceae, Alicante, España.

Key words. Chorology, flora, Orchidaceae, Alicante, Spain.

Como resultado de los estudios que se vienen realizando en los últimos años sobre las orquídeas de la provincia de Alicante, se aportan nuevas localidades de táxones críticos 